\title{
Indications for computed tomography (CT-) diagnostics in proximal humeral fractures: a comparative study of plain radiography and computed tomography
}

\author{
Christian Bahrs' ${ }^{1}$, Bernd Rolauffs*1, Norbert P Südkamp ${ }^{2}$, Hagen Schmal ${ }^{2}$, \\ Christoph Eingartner ${ }^{4}$, Klaus Dietz ${ }^{3}$, Philippe L Pereira ${ }^{5}$, Kuno Weise ${ }^{1}$, \\ Erich Lingenfelter ${ }^{6}$ and Peter Helwig ${ }^{2}$
}

\begin{abstract}
Address: ${ }^{1}$ Klinik für Unfall- und Wiederherstellungschirurgie, BG-Unfallklinik Tübingen, Eberhard-Karls-Universität Tübingen, Schnarrenbergstr. 95, 72076 Tübingen, Germany, ${ }^{2}$ Department für Orthopädie und Traumatologie, Albert-Ludwigs-Universität Freiburg, Hugstetter Str. 55, 79106 Freiburg, Germany, ${ }^{3}$ Institut für Medizinische Biometrie, Eberhard-Karls-Universität Tübingen, Westbahnhofstr. 55, 72070 Tübingen, Germany, ${ }^{4}$ Caritas-Krankenhaus Bad Mergentheim, Klinik für Unfall- und Wiederherstellungschirurgie, Uhlandstr. 797980 Bad Mergentheim, Germany, ${ }^{5}$ Radiologische Klinik, Abt. für Diagnostische und Interventionelle Radiologie Universitätsklinikum Tübingen, Hoppe-Seyler-Straße 3, 72076 Tübingen, Germany and ' Northland Bone and Joint Institute, 2750 Clay Edwards Dr. 304, Kansas City, MO 64116, USA

Email: Christian Bahrs - C.Bahrs@gmx.de; Bernd Rolauffs* - berndrolauffs@yahoo.de; Norbert P Südkamp - norbert.suedkamp@uniklinikfreiburg.de; Hagen Schmal - hagen.schmal@uniklinik-freiburg.de; Christoph Eingartner - Christoph.Eingartner@ckbm.de; Klaus Dietz - klaus.dietz@uni-tuebingen.de; Philippe L Pereira - philippe.pereira@med.uni-tuebingen.de; Kuno Weise - kweise@bgutuebingen.de; Erich Lingenfelter - erichlingenfelter@yahoo.com; Peter Helwig - peter.helwig@uniklinik-freiburg.de

* Corresponding author
\end{abstract}

Published: 2 April 2009

BMC Musculoskeletal Disorders 2009, 10:33 doi:10.1 | 86/ | 47 |-2474-10-33

This article is available from: http://www.biomedcentral.com/I47I-2474/I0/33

(C) 2009 Bahrs et al; licensee BioMed Central Ltd.

This is an Open Access article distributed under the terms of the Creative Commons Attribution License (http://creativecommons.org/licenses/by/2.0), which permits unrestricted use, distribution, and reproduction in any medium, provided the original work is properly cited.

\begin{abstract}
Background: Precise indications for computed tomography (CT) in proximal humeral fractures are not established. The purpose of this study was a comparison of conventional radiographic views with different CT reconstructions with $2 \mathrm{D}$ and $3 \mathrm{D}$ imaging to establish indications for additional CT diagnostics depending on the fractured parts.

Methods: In a prospective diagnostic study in two level I trauma centers, 44 patients with proximal humeral fractures were diagnosed with conventional X-rays (22 AP + axillary views, $22 \mathrm{AP}+$ scapular Y-views) and CT (multi-planar reconstruction (MPR) and maximum intensity projection (MIP)) with $2 \mathrm{D}$ and $3 \mathrm{D}$ imaging. 3 observers assessed the technical image quality, the assessment of the relevant anatomical structures (2-sample-t-test) and the percentage of the osseous overlap of the proximal humerus (Welch-test) using a scoring system. The quality of the different diagnostic methods was assessed according to the number of fractured parts (Bonferroni-Holm adjustment).

Results: There was significantly more overlap of the fractured region on the scapular $Y$-views (mean $71.5 \%$, range $45-$ $90 \%$ ) than on axillary views (mean $56.2 \%$, range 10.5-100\%). CT-diagnostics allowed a significantly better assessment of the relevant structures than conventional diagnostics $(p<0.05)$ independently of the fracture severity (two-, three-, and four-part fractures).

Conclusion: Conventional X-rays with AP view and a high-quality axillary view are useful for primary diagnostics of the fracture and often but not always show a clear presentation of the relevant bony structures such as both tuberosities, the glenoid and humeral head. CT with thin slices technology and additional $3 \mathrm{D}$ imaging provides always a clear presentation of the fractured region. Clinically, a CT should be performed - independently of the number of fractured parts - when the proximal humerus and the shoulder joint are not presented with sufficient $\mathrm{X}$-ray-quality to establish a treatment plan.
\end{abstract}




\section{Background}

After physical examination plain X-rays are essential for diagnostic evaluation of proximal humeral fractures. High-quality radiological diagnostics is based on correct exposure, presentation of the shoulder joint in two views perpendicular to each other with minimal overlap of the fractured region by surrounding osseous structures and soft tissue [1]. Only if these requirements are met, an adequate evaluation of the individual topography, severity and direction of displacement of the fracture is possible, and a reliable classification of the fracture can be performed.

In particular, a computed tomography (CT) is recommended for complex fracture situations although those situations were not clearly defined. Therefore, precise indications for CT in proximal humeral fractures are not established. In addition, its benefit remains unclear [2-6].

A review of the literature showed that most often the anteroposterior view (AP view) and the scapular Y- view and the axillary view are used for routine diagnostics [7$11]$.

Various modifications of the axillary view of the shoulder are described [12-15]. The shoulder joint lies between the sagittal and coronal plane of the body and therefore correct radiological presentation is difficult[16]. Interpretation of the X-rays is impaired by multiple fracture lines of the usually complex injury[5]. The purpose of this study was a comparison of conventional radiographic views (AP view, scapular Y-view, axillary view) and the CT diagnostics (using either MIP or MPR reconstructions) in twodimensional (2 D) and three-dimensional (3 D) visualization with either on film or individual on-screen presentation. Special attention was given to the technical quality of the method, osseous overlapping and assessment of relevant anatomical structures of the shoulder joint in proximal humeral fractures. We also wanted to establish precise indications for CT diagnostics depending on the part analysis according to the Neer classification.

\section{Methods}

The study was performed with IRB approval in two university trauma centers. In this prospective study a consecutive series of 44 patients with acute proximal fractures that presented to the two university centers were included. No patients were excluded during this time period from the study.

In the first center 22 patients with a proximal humeral fracture (16 female/ 6 male, median age 64 years, range 36-94 years) were diagnosed with conventional analogous plain radiography with an AP view $(66-70 \mathrm{kV} / 12.5-$ $16 \mathrm{mAs}$ ) with the patient standing and the arm extended and an axillary view (66-70 KV/5-8 mAs) with the patient sitting and a minimum of $60^{\circ}$ abduction of the arm in analogous technology and automatic exposure (Polydoros $50 \mathrm{~s}^{\circledast}$, Siemens, Erlangen, Germany). Afterwards a standardized CT with the patient supine (Somatom Sensation $16^{\oplus}$, Siemens, Erlangen, Germany) with MPR reconstruction of the data set and $2 \mathrm{D}$ and $3 \mathrm{D}$ imaging was performed. 2 D CT was carried out with a slice thickness of $0.75 \mathrm{~mm}$ in the osseous window. The $3 \mathrm{D}$ reconstruction was performed with $1 \mathrm{~mm}$ layers. Films with 60 images for the 2 D CT and 12-15 pictures for 3 D reconstructions were printed and provided for viewing.

In the second center, for 22 patients with a proximal humeral fracture ( 17 women $/ 5$ men, median age 73 years, range 36-84 years), conventional digital radiography was applied with an AP view and a scapular Y-view with the patient standing (Device Polydoros Sx 50 ${ }^{\circledast}$, Siemens, Erlangen, Germany, Tube Optilix 150/30/50 C, Memory Foils System PCR Eleva Philips Electronics ${ }^{\circledast}$, Hamburg, Germany).

Then, a $2 \mathrm{D}$ and $3 \mathrm{D}$ presentation after MIP reconstruction of the dataset (Siemens sensation $64^{\oplus}$, Erlangen, Germany) with automatic individual adaptation was provided. Utilizing special software equipment, it was possible to assess each section of the CT-scans individually and from different directions with on-screen workstations. 2 D CT was carried out with a slice thickness of 0.75 $\mathrm{mm}$ in the osseous window. The $3 \mathrm{D}$ reconstruction was performed with $1 \mathrm{~mm}$ layers. In general, 60 pictures for the $2 \mathrm{D}$ CT and $12-15$ for the 3 D-reconstructions were analyzed. The different radiographic views and CT techniques, different viewing methods, and different processing methods were used at each center, and therefore each center was considered as a separate group and both centers were compared with each other. Nevertheless, the consistencies of some AP views of the two centers were analyzed and were comparable.

All conventional X-rays were collected, scanned and analyzed with a processing program AutoCAD $2000^{\oplus}$ (Autodesk GmbH Munich, Germany). According to the literature we defined the area of the proximal humerus as the square of the longest diameter of the epiphysis[17].

The percentage of the overlap surface of the proximal humerus by the surrounding osseous structures (acromion, lateral clavicula, coracoid, glenoid) were calculated and documented for the AP view, scapular Y- and axillary views.

Because of multi-planar visualization of the proximal humerus, there was no overlap in CT diagnostics. 
All cases were presented to three investigators who were not involved in the care of the patients. Observer 1 was an experienced orthopaedic surgeon with fellowship training in shoulder surgery. Observer 2 was an experienced trauma surgeon with a special interest in bone and joint orthopaedic surgery. Observer 3 was a fellow for trauma surgery with a special interest in bone and joint surgery. The investigators independently evaluated the three imaging methods (conventional X-ray, 2 D CT, 3 D CT) for all 44 patients. All available images were used for evaluation of the relevant structures. They scored the technical quality of each of the two views of the conventional X-rays and the CT-scans separately. According to an imaging analysis score of Leschka et. al. and the European guidelines on quality criteria for diagnostic radiographic images we defined the essential parameters for the assessment as film density, contrast and sharpness of the relevant structures. The used scoring system consisted of 4 grades $(1=$ excellent, 2 = good, 3 = fair, and $4=$ inadequate) $[18,19]$. For the assessment of the relevant structures the following five structures were defined to be relevant:

a) the greater tuberosity (on AP view), b) the glenoid and the humeral head (on AP view and axillary view/scapular Y-view), c) the coracoid (on axillary view/scapular Yview), d) the lesser tuberosity (on axillary view/scapular Y-view) and e) the subacromial space (on AP view).

The essential parameters for the analysis of the relevant structures were the complete and clear presentation, the ability to estimate the degree of comminution and the degree of displacement. The scoring system consisted again of 4 grades (see above).

Average scores over all three investigators and two views were calculated for conventional and CT-diagnostics for assessment of technical quality and identifiability of relevant anatomical structures.

44 fractures were classified according to the part analysis of the Neer classification during a consensus conference of the three observers, who were not involved in patient care $[20,21]$.

The statistical calculations were carried out with the statistics program JMP 6 (SAS campus drive, Building S., Cary, NC, 27513 SAS Institutes, Cary, NC, USA)

Frequencies of nominally scaled characters (part analysis of the Neer classification) were compared by a contingency table analysis with the chi square test. Averages of continuous variables were compared with the 2-sample-ttest, if the variance of the distributions did not differ significantly. For descriptive statistics we used the arithmetic means together with their $95 \%$ confidence intervals. For unequal variances the Welch-test was used. (E.g., proportion of overlap of the proximal humerus.) For multiple tests we used a Bonferroni-Holm adjustment of the significance level. For other statistical tests then the BonferroniHolm adjustment, the significance level was $\rho<0,05$.

\section{Results \\ Classification}

In the first center there were twelve 2-part, seven 3-part and three 4-part fractures and in the second center there were nine 2-part, eight 3-part and five 4-part fractures. The whole group enclosed twenty-one 2-part, fifteen 3-part and eight 4-part fractures.

With regard to the part analysis of the Neer classification there were no significant differences between the centers. $(\rho=0.60)$

\section{Technical image quality}

The mean quality scores for the three methods and the two centers are exhibited with their $95 \%$ confidence limits in Fig. 1. The quality of the conventional X-rays (AP and axillary views) carried out in analogous technology in center 1 was significantly worse than the X-rays made in digital technology (AP and scapular Y-view) in center 2. ( $\rho$ $<0.05$ ) There was no significant difference in quality of $\mathrm{X}$ -

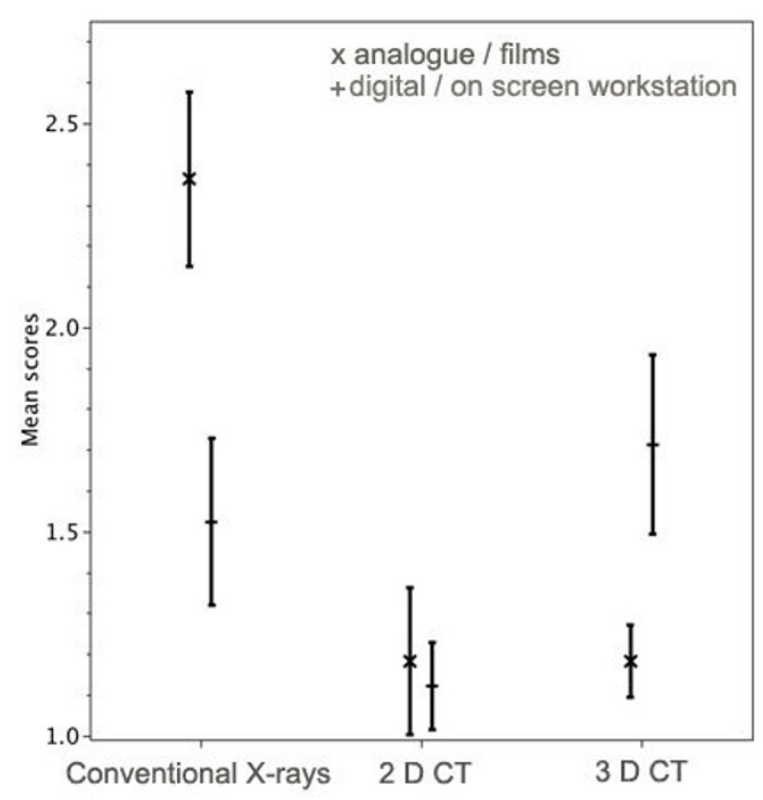

\section{Figure I}

Technical image quality. The mean quality scores for the three methods (Conventional X-ray; 2 D CT, 3 D CT) and the two methods of presentations ( $x=$ analogue/films, $+=$ digital/workstation) are shown with their $95 \%$ confidence intervals. 
rays made in digital technology and quality of $3 \mathrm{D} C \mathrm{CT}$ images on a workstation. $(\rho>0.05)$ The quality of the $3 \mathrm{D}$ CT images individually viewed on a workstation was significantly worse than the 3 D CT presented on films. $(\rho<$ $0.05)$ There were no differences between the qualities of the 2 D CT techniques. $(\rho>0.05)$

\section{Osseous overlap of the conventional X-ray diagnostics}

There was significantly more osseous overlap of the fractured region on the scapular Y-views than on axillary views. $(\rho<0.05)$ (Fig. 2$)$. The scapular Y-views showed a median overlap of $71.5 . \%$ (range $45-90 \%$ ). The median overlap of all axillary views was smaller with $56.2 \%$ but a much larger range (10.5-100\%). The median overlap of the AP view was only $5.8 \%$ (range from 0 to $26 \%$ ). For the AP view, there was no significant difference between the two centers $(\rho=0.7)$.

\section{Assessment of relevant structures of the shoulder joint and the proximal humerus}

The relevant structures were graded significantly better on 2 D CT with individual visualization on a workstation than on 2 D CT presented on films $(\rho<0.05) .3$ D viewed on films was significantly better than $3 \mathrm{D}$ viewed on workstations. $(\rho<0.05)$ In addition, significant differences between the conventional radiographs and the $2 \mathrm{D} C \mathrm{CT}$ and 3 D CT-investigations were found. $(\rho<0.05)$ (Fig 3 )

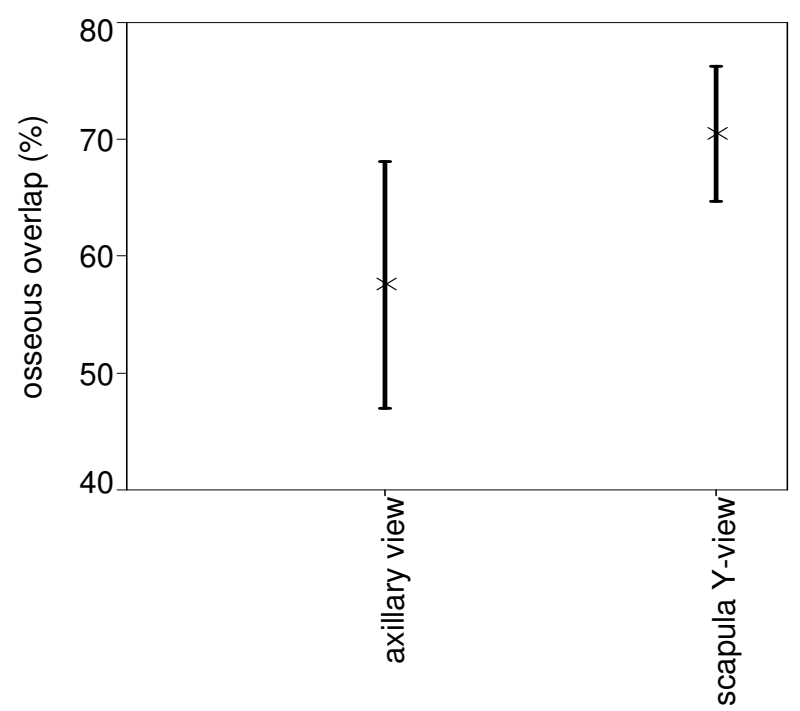

Figure 2

Percentage of osseous overlap of conventional X-ray diagnostics (axillary view and scapular-Y-view) with their $\mathbf{9 5 \%}$ confidence intervals. Due to different variances in the two samples, the confidence intervals are different.

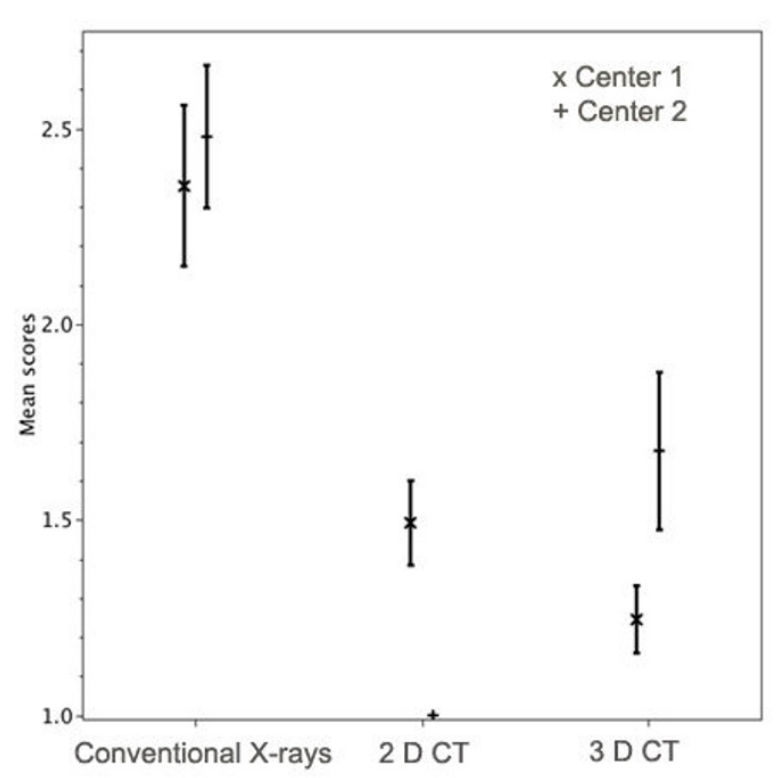

Figure 3

Assessment of relevant structures of the shoulder joint and the proximal humerus. The mean quality scores for the three methods and the two centers are shown with their $95 \%$ confidence intervals.

Neer classification for assessment of relevant structures of the shoulder joint and the proximal humerus

For all fracture severities - 2-, 3-, or 4-part fractures - and independently of the two centers, CT-diagnostics were significantly better than conventional radiographic diagnostics $(\rho<0.05)$. 2 D CT-diagnostics with image presentation on workstation were the best modality (Fig. 4)

\section{Discussion}

A comparison of the different diagnostic methods with regard to technical quality, osseous overlapping and assessment of the relevant structures has not been presented in proximal humeral fractures. With the present investigation we tested various common conventional Xray-views and CT in $2 \mathrm{D}$ and $3 \mathrm{D}$ imaging in proximal humeral fractures.

Our study showed a significantly better technical image quality of digital radiographs compared to analogous radiographs. In addition, 3 D CT reconstructions presented on films were better than 3 D CT reconstructions viewed on workstation. Furthermore, the digital conventional image quality was equal to the quality of the 3 D CT on workstation. Therefore, the quality standard, of the digital conventional radiography possibly was of such a high level that it reached the quality of a technically superior method such as $3 \mathrm{D}$ CT. Those results may be related 


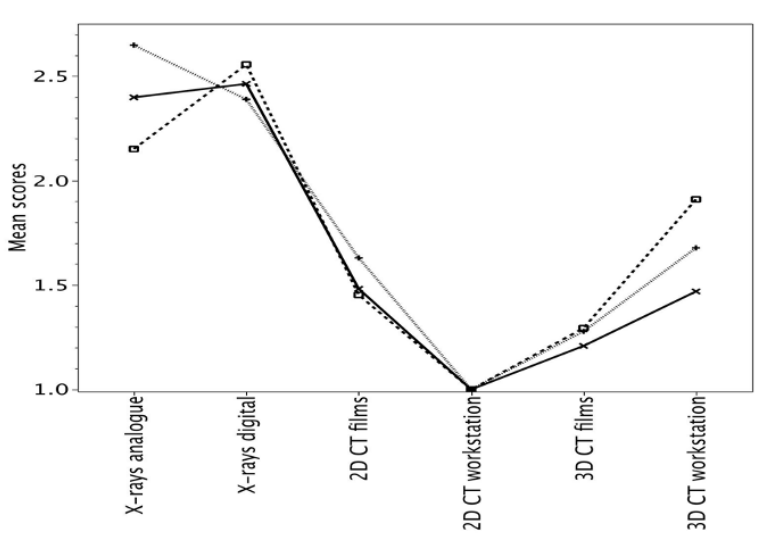

Figure 4

Assessment of relevant structures of the shoulder joint and the proximal humerus according to fractured parts (2-parts $=$ x, 3-parts $=\square$, 4-parts $=+$ ). The mean quality scores for the three methods and the two centers are shown.

to a center-specific technical quality standard and the technical availability of modern diagnostic equipment and the expertise in digital image acquisition and processing.

Comparing the osseous overlap in different conventional radiographic views, our study showed that there was 15\% less overlap of the fractured region by surrounding osseous structures on the axillary views than on the scapular Y-view. This resulted in a significant difference in the assessment of the relevant structures. Despite the importance of image quality, the choice of an appropriate view to minimize osseous overlap (e.g. axillary view) was found to be of equal importance for the visualization of the relevant structures.

The plain X-rays are still the most important tool for initial fracture diagnostics. In the commonly used trauma series the gold standard for initial evaluation is an AP. view in the plane of the scapula, a scapular Y- and the axillary view. According to Neer, for the initial evaluation of proximal humeral fractures an AP- view and a scapular Yview are recommended. If the fracture visualization remains unclear, an axillary view is also necessary [21]. Our results demonstrate the superiority of the axillary view in overlap and the assessment of the relevant structures, when compared to the scapular Y-view. Similar to our study, other authors also recommend strictly the axillary view as a standard in combination with an AP view [11,22-24].

Sidor et al. classified 50 proximal humeral fractures with the help of the trauma series (AP, axillary, scapular Y- view). They examined the views of the trauma series according to their contribution of information for fracture classification. They showed that a correct classification of the fracture was possible by combination of AP view and axillary view in $99 \%$ of the cases. A combination of AP view and scapular Y-view resulted only in $79 \%$ of the cases in a correct classification. They concluded that an axillary view delivers significantly more information about fracture classification than the scapular Y-view [25].

The conduction of the AP view is usually not associated with technical problems. The AP view delivers an almost overlapping free visualization of the proximal humerus. Usually, an adequate assessment of relevant structures such as the greater tuberosity, glenoid and subacromial space is possible in most of the cases. Difficulties originate when X-rays are taken with the arm in internal rotation and when an inclination of the X-ray beam is chosen which is unsuitable to show the joint gap. Therefore, a posterior dislocation could be missed due to an overlap of the humeral head and the glenoid [23,26-28].

The scapular Y-view as a second view permits the assessment of the position of the joint and the relationship between the shaft and head of the humerus. However, we found an average osseous overlap of the proximal humerus and shoulder joint of about $70 \%$. In addition, the evaluation of relevant structures, especially of the glenoid and the humeral head and the lesser tuberosity was considerably limited.

For the axillary view, an abduction of the arm is mandatory and therefore painful. The performance is technically demanding and difficult to reproduce. Although we have significantly better technical image quality in the digital than the analogous radiographs, we could show that the analogous axillary view of the fractured region resulted in significantly less osseous overlap of $56 \%$ than the digital scapula-y image $(72 \%)$. Various modifications of this view were described [12-15]. However, it is common that the abduction may not be performed and the X-ray-evaluation may be limited. However, this was not a problem in the current study. Simon and co-authors evaluated axillary views on standardized osteotomized proximal humeri with different arm positions (in flexion, extension, abduction). They found, that in $30^{\circ}$ of abduction of the arm, the real displacement of the fracture was not reproducibly represented on axillary views. Only if the arm is held in roughly $60^{\circ}$ to $90^{\circ}$ abduction in neutral position, reproducible results of the fracture displacement can be expected [29]. In our study all axillary views were performed with the patient sitting and 60-70 abduction of the arm with neutral rotation. With this approach a good assessment of the fractured proximal humerus, the head-shaft-axis, the position of the shoulder joint was 
possible and therefore adequate information was provided.

We could prove that even in simple fractures such as 2part fractures according to Neer, the relevant bony structures of the humerus and shoulder joint were poorer to judge in the conventional X-rays than in the CT-scans. By multi-plane visualization of the fractured region in $2 \mathrm{D}$ technology and 3 D-reconstruction of the dataset, the complete overlapping-free presentation is the most important advantage of this technique. All investigators graded the 2 D CT-scans as the best modality for diagnostics. In terms of technical image quality and presentation of the relevant structures, regardless of the fracture complexity, we found that the CT-diagnostics were better than the conventional X-rays. Various authors stated that the CT is a helpful diagnostic tool for evaluation of proximal humeral fractures. Nevertheless, those reviews included recommendations without exact definition of their use $[2,3,30]$. Because the visualization could be further improved by using a thinner layer thickness up to 0.75 $\mathrm{mm}$, this method is now more often used for diagnostics in proximal humeral fractures. However, clear indications when to use a CT are not yet defined.

\section{Conclusion}

If image quality impairs fracture visualization or if osseous overlap prevents the visualization of the fractured structures, conventional radiography is not sufficient. In such a situation, we believe that a CT should be performed.

\section{Competing interests}

The authors declare that they have no competing interests.

\section{Authors' contributions}

$\mathrm{CB}$ designed, coordinated and conceived the study, acquisition and analysis of data, wrote and corrected the manuscript. HS. participated in evaluation of radiographs and CT scans, corrected the manuscript and approved the final manuscript. EL participated in evaluation of radiographs and CT scans, corrected the manuscript and approved the final manuscript. BR coordinated and conceived the study, analysis of data, drafted the manuscript, revised the manuscript critically for important intellectual content. CE, NPS, PLP and KW revised the manuscript critically for important intellectual content and approved the final manuscript KD performed the statistical analysis, corrected the manuscript and approved the final manuscript. $\mathrm{PH}$ participated in evaluation of radiographs and CT scans, revised the manuscript critically and approved the final manuscript

\section{Acknowledgements}

We want to thank Mrs. L. Wellhäuser and the staff of the Department of Diagnostic Radiology in the BG Trauma Center Tuebingen and the university hospital of Freiburg for obtaining the radiographs and the CT scans.

\section{References}

I. Nicholson DA, Lang I, Hughes P, Driscoll PA: ABC of emergency radiology. The shoulder. Bmj 1993, 307(69 | 2): I I29-I I 34 .

2. Billet FP, Schmitt WG, Gay B: Computed tomography in traumatology with special regard to the advances of threedimensional display. Arch Orthop Trauma Surg 1992, I I I(3): $|3|-\mid 37$.

3. Castagno AA, Shuman WP, Kilcoyne RF, Haynor DR, Morris ME, Matsen FA: Complex fractures of the proximal humerus: role of CT in treatment. Radiology 1987, 165(3):759-762.

4. Kuhlman JE, Fishman EK, Ney DR, Magid D: Two-and threedimensional imaging of the painful shoulder. Orthop Rev 1989 , I8(II): | $20|-| 208$.

5. Sidor ML, Zuckerman JD, Lyon T, Koval K, Cuomo F, Schoenberg N: The Neer classification system for proximal humeral fractures. An assessment of interobserver reliability and intraobserver reproducibility. J Bone Joint Surg Am 1993, 75(1 2): 1745-1750.

6. Siebenrock KA, Gerber C: The reproducibility of classification of fractures of the proximal end of the humerus. J Bone Joint Surg Am 1993, 75(1 2): I75I-1755.

7. lannotti JP, Ramsey ML, Williams GR Jr, Warner JJ: Nonprosthetic management of proximal humeral fractures. Instr Course Lect 2004, 53:403-416.

8. Neer CS 2nd: Four-segment classification of proximal humeral fractures: purpose and reliable use. J Shoulder Elbow Surg 2002, I I (4):389-400.

9. Lill $\mathrm{H}$, Josten $\mathrm{C}$ : [Proximal and distal humerus fractures in advanced age]. Orthopade 2000, 29(4):327-34I.

10. Szyszkowitz R, Schippinger G: [Fractures of the proximal humerus]. Unfallchirurg $1999,102(6): 422-428$.

II. Resch H: [Fractures of the humeral head]. Unfallchirurg 2003, 106(8):602-617.

12. Geusens $E$, Pans S, Verhulst D, Brys P: The modified axillary view of the shoulder, a painless alternative. Emerg Radiol 2006, I 2(5):227-230.

13. Bernageau J, Faguer B, Debeyre J: [Arthropneumotomographic study of a case of recurring dislocation of the shoulder]. Rev Rhum Mal Osteoartic 1966, 33(3): I35-137.

14. Clements RW: Adaptation of the technique for radiography of the glenohumeral joint in the lateral position. Radiol Technol |979, 5 I (3):305-3 |2.

15. Putkonen M, Lahde S, Puranen J, Paivansalo M: The value of axial view in the radiography of shoulder girdle - experiences with a new modification of positioning. Rontgenblatter 1988, 4I(4): I58-162.

16. Richardson JB, Ramsay A, Davidson JK, Kelly IG: Radiographs in shoulder trauma. J Bone Joint Surg Br 1988, 70(3):457-460.

17. Murphy W, Leu D: Fracture Classification: biological significance. In AO Principles of Fracture Management Volume I. first edition. Edited by: Rüedi TP, Murphy WM. Stuttgart/New York: Thieme; 2000:45-58.

18. Leschka S, Alkadhi H, Boehm T, Marincek B, Wildermuth S: Coronal ultra-thick multiplanar CT reconstructions (MPR) of the pelvis in the multiple trauma patient: an alternative for the initial conventional radiograph. Rofo 2005, I77(10): I 405-I4II.

19. JHE Carmichael CM, Moores BM, Oestmann JW, Schibilla H, Teunen RVTaBW D: European Commission EUR 16260 - European Guidelines on Quality Criteria for Diagnostic Radiographic Images. Luxembourg, Office for Official Publications of the European Communities 1996, VIII:80.

20. Neer CS 2nd: Displaced proximal humeral fractures. I. Classification and evaluation. J Bone Joint Surg Am 1970, 52(6): $1077-1089$.

21. Neer CS 2nd: Displaced proximal humeral fractures: part $I$. Classification and evaluation. 1970. Clin Orthop Relat Res 2006, 442:77-82.

22. Schnabel M, Bahrs C, Walthers E, Gotzen L: [Marburg shoulder radiography splint (MSR splint) for standardized and high 
quality plain film radiography in fractures of the proximal humerus]. Unfallchirurg 2004, 107( I I): 1099-1 102.

23. Clough TM, Bale RS: Bilateral posterior shoulder dislocation: the importance of the axillary radiographic view. Eur J Emerg Med 200I, 8(2): $161-163$.

24. Heyer D: [Imaging methods in traumatology of the shoulderand elbow joint]. Aktuelle Radiol 1996, 6(5):225-23I.

25. Sidor ML, JD Z, T L, K K, N S: Classification of proximal humerus fractures: The contribution of the scapular lateral and axillary radiographs. Journal of Shoulder an Elbow Surgery 1994, 3(I):24-27.

26. Espag MP, Back DL, Baroni M, Bennett AR, Peckham TJ: Diagnosing shoulder dislocations: time for a change of view. Ann $R$ Coll Surg Engl 2002, 84(5):334-337.

27. Hatzis N, Kaar TK, Wirth MA, Rockwood CA Jr: The often overlooked posterior dislocation of the shoulder. Tex Med 200I, 97(I I):62-67.

28. Perron $A D$, Jones RL: Posterior shoulder dislocation: avoiding a missed diagnosis. Am J Emerg Med 2000, 18(2): I89-191.

29. Simon JA, Puopolo SM, Capla EL, Egol KA, Zuckerman JD, Koval KJ: Accuracy of the axillary projection to determine fracture angulation of the proximal humerus. Orthopedics 2004, 27(2):205-207.

30. Kuhlman JE, Fishman EK, Ney DR, Magid D: Complex shoulder trauma: three-dimensional CT imaging. Orthopedics 1988, II(II):I56I-I563.

3I. Hertel R, Hempfing A, Stiehler M, Leunig M: Predictors of humeral head ischemia after intracapsular fracture of the proximal humerus. J Shoulder Elbow Surg 2004, I 3(4):427-433.

\section{Pre-publication history}

The pre-publication history for this paper can be accessed here:

http://www.biomedcentral.com/1471-2474/10/33/pre

pub
Publish with Bio Med Central and every scientist can read your work free of charge

"BioMed Central will be the most significant development for disseminating the results of biomedical research in our lifetime. "

Sir Paul Nurse, Cancer Research UK

Your research papers will be:

- available free of charge to the entire biomedical community

- peer reviewed and published immediately upon acceptance

- cited in PubMed and archived on PubMed Central

- yours - you keep the copyright 